\title{
PERSONAS EN SITUACIÓN DE DISCAPACIDAD VISUAL EN RELACIÓN A LAS BARRERAS Y ESTRATEGIAS QUE AFECTAN LAS ACTIVIDADES DE LA VIDA DIARIA INSTRUMENTALES
}

\author{
PERSONS WITH VISUAL IMPAIRMENT IN RELATION WITH BARRIERS AND \\ STRATEGIES AFFECTING THEIR PERFORMANCE IN INSTRUMENTALS DAILY LIVING \\ ACTIVITIES
}

\section{Carla Alarcón S. ${ }^{1}$ y María Vizcarra Q. ${ }^{2}$}

\begin{abstract}
Resumen
El objetivo del estudio de metodología cuantitativa fue conocer la información disponible sobre el desempeño en las actividades de la vida diaria instrumentales (AVDI) de las personas en situación de discapacidad visual en relación a las barreras que presentan para su ejecución. Para ello se llevó a cabo una revisión bibliográfica en distintas bases de datos y sitios web y documentos e información disponible en todo tipo de prensa. Las variables utilizadas fueron: actividades de la vida diaria instrumental, discapacidad visual, barreras, edad, año, idioma y país.

Del total de la información recopilada, el 32,60 \% de las investigaciones tenían relación con la temática a investigar. En su mayoría correspondía a publicaciones del año 2008 con un 17,4\%, siendo con mayor frecuencia documentos en idioma español (78,3\%). En la literatura de Chile se encontró aproximadamente un 21,7\% de información.

La mayoría de la literatura incluía a todas las personas en situación de discapacidad con un porcentaje de 58,7\%, todas éstas relacionadas con las barreras y AVDI, sin embargo, los documentos abarcaban mayor información sobre las barreras de comunicación $(32,6 \%)$ y las AVDI relacionadas con la gestión de comunicación (37\%). En conclusión la información para aquellas actividades como el cuidado de mascotas y crianza de niños y las investigaciones sobre el tema abordado, es insuficiente para así poder implementar estrategias que faciliten la independencia de estas personas.
\end{abstract}

\section{Palabras claves}

Discapacidad Visual, Actividades de la Vida Diaria Instrumentales, Barreras ArquitectónicasAbstract

The aim of this research with quantitative methodology was to know the available information about the performance at instrumental daily life activities of people with visual impairment in relation to the barriers that present to their performance. For this, a bibliographic review was carried out in different databases and websites, documents and information available in all types of press. The variables used were instrumental activities of daily life, visual impairment, barriers, age, year, language and country.

The total of the information gathered, 32.60 per cent of investigations had related to theme to investigate.

The greatest amount of information found corresponded to publications of year 2008 with 17.4 per cent, being more frequently documents of Spanish language (78.3\%). In the Chilean literature, approximately 21.7 per cent of information was found. Most of information included all persons in situations of disability with a percentage of 58.7 per cent, all these related to barriers and IADL however, documents included more information on the communication barriers(32.6 per cent) and IADL related to the management of communication (37 per cent).

In conclusion, the information for activities such as pet care, child care and researching on the main theme is insufficient to implement strategies that facilitate the independence of these persons.

\section{Keywords:}

Visual disability, Instrumentals Activities of Daily Living, Architectural Barriers.

Fecha de recepción: 19/07/2016

Fecha aceptación: 15/11/2016

1 Licenciada en ciencia de la ocupación. Universidad Santo Tomás Concepción. 4030000. +56965803878. Carlaalarcon42@alumnos.cst Licenciada en ciencia de la ocupación. Universidad Santo Tomás Concepción. 4030000. +56978950305. Mariavizcarra993@gmail.com 


\section{INTRODUCCIÓN}

Según la American Occupational Therapy Association, (2010) la ocupación es toda actividad significativa para la persona, tiene un propósito y un fin (Ávila, 2010). De acuerdo al Marco de Trabajo para la Práctica de Terapia Ocupacional las ocupaciones están clasificadas en: actividades de la vida diaria básicas (AVDB), actividades de la vida diaria instrumentales (AVDI), educación, ocio y tiempo libre, sueño y descanso, participación social, trabajo y juego. El ser humano participa en ocupaciones de acuerdo a su ciclo vital permitiéndole diversidad de experiencias, integrarse a la sociedad y experimentar distintos niveles de satisfacción personal. Las personas con discapacidad pueden ver limitadas la realización de sus tareas cotidianas producto del entorno en cual están insertos. Uno de estos factores pueden ser los obstáculos existentes en el entorno y la poca accesibilidad de los diversos espacios ya sean públicos o privados (Lorenzo, 2005).

La discapacidad es un término genérico que incluye deficiencias de las funciones y/o estructuras corporales, limitaciones en la actividad y restricciones en la participación, indicando los aspectos negativos de la interacción entre un individuo (con una "condición de salud") y sus factores contextuales (factores "ambientales y personales") (Organización Mundial de la Salud, 2001).

El estudio nacional de la discapacidad en Chile (ENDISC Chile, 2004) expresa que el $12,9 \%$ de la población chilena presenta algún tipo de discapacidad, lo que equivale a 2.068.072 personas (FONADIS, 2004). Por otro lado, según algunos avances de los resultados del ENDISC II en Chile un 20\% de las personas mayores de 18 años presentan algún tipo de discapacidad, es decir, 2.606.914 de personas (Gobierno de Chile, 2016). Este número supera a dicha población con un $13 \%$, en relación con el estudio anterior (Lagos, 2016). Mediante estos datos podemos observar que dicha población ha ido en aumento, lo que es corroborado por el Estudio Nacional de Discapacidad donde se observó un aumento en porcentaje en las personas que padecen de alguna discapacidad través del tiempo

El foco de este estudio será solo en personas que presentan alguna situación de discapacidad visual. A nivel mundial aproximadamente 285 millones de per- sonas presenta una discapacidad visual, de las cuales 39 millones son ciegas y 246 millones presentan baja visión (Organización Mundial de la Salud, 2014). En Chile las personas con discapacidad visual representan el $11,9 \%$ de la población (Ministerio de Desarrollo Social, 2016).

De acuerdo a lo anterior se puede inferir que las personas que presentan discapacidad visual representan un porcentaje no menor en la población, sin embargo, debemos destacar que la información disponible y los datos para dicha población es muy reducida, si bien la sociedad ha implementado nuevas estrategias para mejorar la accesibilidad de las personas en situación de discapacidad y fomentar su participación, inclusión social y calidad de vida sin importar su condición, estos implementos van enfocados hacia personas con discapacidad física, dejando de lado a una población que presenta gran porcentaje de discapacidad en nuestro país y en el mundo que son las personas en situación de discapacidad visual (Boudeguer, Prett y Squella, 2010).

La investigación desarrollada busca conocer aquella información disponible en el desempeño de las actividades de la vida diaria instrumentales (AVDI) de las personas en situación de discapacidad visual, en relación a las barreras y estrategias disponibles.

\section{Metodologia}

Se llevó a cabo una revisión bibliográfica con análisis cuantitativo de tipo descriptivo. La temática a investigar está relacionada con el desempeño de las AVDI de las personas en situación de discapacidad visual y con las barreras que estas presentan para su ejecución. La búsqueda de datos fue a través de diversas fuentes de información como revistas, manuales, noticias, sitios web y estudios, con evidencia o sin evidencia científica desarrolladas a través de los años.

Las palabras claves usadas en la búsqueda fueron: discapacidad visual, desempeño en las actividades de la vida diaria instrumentales y barreras, tanto en los idiomas; español e inglés, utilizando además los sinónimos de estas palabras (tabla 1) 
Tabla 1:

Variables

\begin{tabular}{|c|c|}
\hline Español & Inglés \\
\hline Discapacidad Visual & Visual Disability \\
Limitación visual & Visual limitation \\
Baja visión & Lowvision \\
Personas no videntes & Blindpeople \\
Ciegos & Blind \\
\hline Actividades de la Vida Diaria Instrumentales & Purchases \\
Compras & Money handling \\
Manejo de dinero & Transport \\
Transporte & Mobilization \\
Movilización & Othercare \\
Cuidado de otros & Instrumental Activities of Daily Living \\
Independencia en comunicación. & Independence in communication \\
Desempeño ocupacional en AVD Instrumentales & Occupational performance in instrumental activities of \\
& daily living \\
\hline Barreras & Obstacles \\
Obstáculos & Accessibility \\
Accesibilidad & Impairments \\
Impedimentos & Limitations \\
Limitaciones & Exclusion \\
Exclusión & \\
& \\
\hline & \\
\hline
\end{tabular}

Los artículos se seleccionaron de acuerdo a las variables que se querían estudiar, utilizando las palabras claves. Por ello, se leyó el resumen de cada documento, estudio o noticia para la selección, una vez seleccionados, y teniendo la cantidad suficiente de bibliografía, se fue leyendo de manera exhaustiva para la inclusión y exclusión de los estudios y así comenzar con el análisis de datos.

Para seleccionar cada artículo se realizaron las siguientes preguntas: ¿Qué actividad instrumental incluía la información?, ¿Cuánta información es del país y cuánta extranjera?, ¿Qué tipo de discapacidad visual se incluye en la información, y qué tipos de barreras?, ¿Cuántas barreras afectan la autonomía e independencia de las personas en situación de discapacidad visual?

\section{Resultados}

La cantidad de información disponible fue de 46 documentos, los cuales abarcan el desempeño de las AVDI de las personas en situación de discapacidad visual y su relación con las barreras que presentan para ejecutarlas

\section{Tipo de estudio:}

De los 46 documentos encontrados y analizados, el mayor porcentaje de información fue el de investigaciones, lo que equivale a un $32,60 \%$, luego noticias con un porcentaje de $28,30 \%$, en tercer lugar encontramos proyectos de investigación representando un $10,9 \%$ del total (Tabla Nº2). 
Tabla $\mathrm{n}^{\circ} 2$ :

Tabla de frecuencia de los tipos de estudios.

\begin{tabular}{|c|c|c|}
\hline & Frecuencia & Porcentaje \\
\hline Tesis de alumnado & 1 & $2,20 \%$ \\
\hline Estudio & 3 & $6,50 \%$ \\
\hline Experiencia & 1 & $2,20 \%$ \\
\hline Guía de Transporte & 1 & $2,20 \%$ \\
\hline Investigación & 15 & $32,60 \%$ \\
\hline Manual & 1 & $2,20 \%$ \\
\hline Noticia & 13 & $28,30 \%$ \\
\hline Página Web & 4 & $8,70 \%$ \\
\hline Proyecto de Implementación & 5 & $10,90 \%$ \\
\hline Revisión Bibliográfica & 1 & $2,20 \%$ \\
\hline Revista & 1 & $2,20 \%$ \\
\hline Total & 46 & $100 \%$ \\
\hline
\end{tabular}

\section{País:}

En relación con la variable país, podemos señalar que mayoritariamente encontramos artículos de España con un 30,4\%, posteriormente encontramos de Chile con $21,7 \%$ de publicaciones relaciones con la temáti$\mathrm{ca}$, mientras que de Argentina, México y Colombia solo encontramos 3 artículos representando un 6,5\% del total (cada uno).
El 28,3\% restante se atribuye a los países de África, Austria, Brasil, Canadá, Estados Unidos, Honduras, India, Noruega, Polonia, y otros artículos de los cuales no se encontraba disponible dicha variable. La mínima frecuencia de artículos encontrados fue de 1 y la frecuencia mayor es de 2 de artículos (Figura №1).

Figura $N^{0}$ 1: Información disponible y realizada en los diversos países.

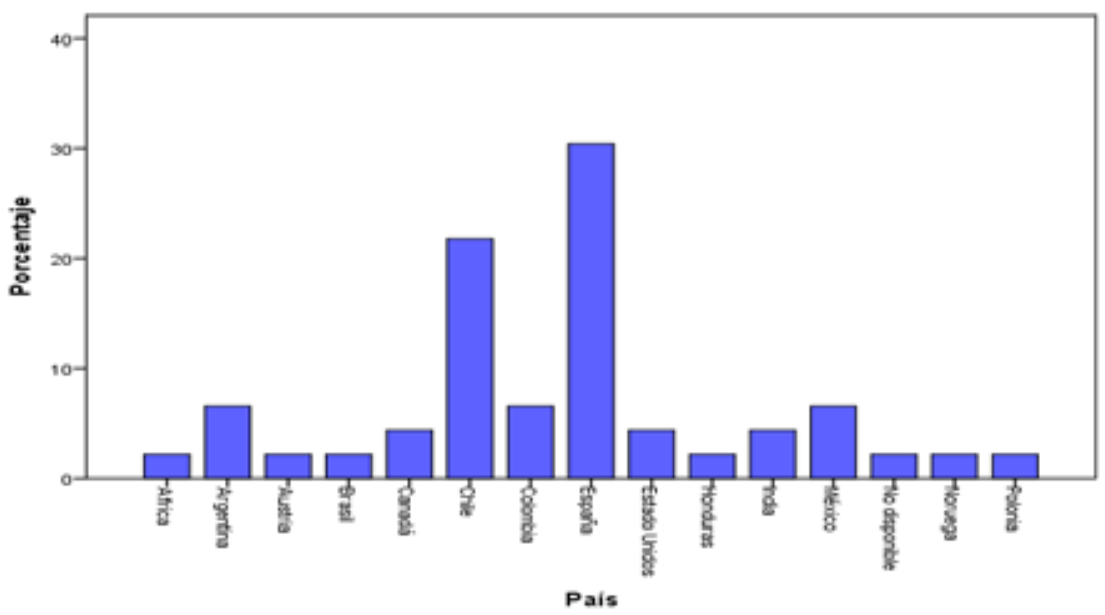




\section{Idioma:}

Se evidencian 36 de artículos, analizados en idioma español, es decir, en un 78,3\%; el segundo idioma más utilizado es el inglés, representando el $21,7 \%$ del total (Tabla No 3).

Tabla No3:

Tabla de frecuencia de idiomas

\begin{tabular}{|c|c|c|}
\hline & Frecuencia & Porcentaje \\
\hline Español & 36 & $78,3 \%$ \\
\hline Inglés & 10 & $21,7 \%$ \\
\hline Total & 46 & $100 \%$ \\
\hline
\end{tabular}

\section{Tipo Discapacidad Visual}

De acuerdo a la información analizada, podemos señalar que el $58.7 \%$ de los artículos incluye a las personas que presentan alguna discapacidad visual considerando las diversas sub clasificaciones existentes (severa, profunda, moderada, ceguera). Seguido de esto, el $32,6 \%$ de los artículos, hacen referencia a la población que presenta ceguera. Por último la frecuencia mínima de información analizada fue la sub clasificación de discapacidad visual severa y profunda con un porcentaje de $6,5 \%$ (Tabla No4).

Tabla No 4:

Tabla de frecuencia de tipos de discapacidad visual

\begin{tabular}{|c|c|c|}
\hline & Número de casos & Porcentaje \\
\hline Moderada & 7 & $15,2 \%$ \\
\hline Severa & 2 & $4,3 \%$ \\
\hline Profunda & 1 & $2,2 \%$ \\
\hline Ceguera & 15 & $32,6 \%$ \\
\hline Todas & 27 & $58,7 \%$ \\
\hline Total & 52 & $100 \%$ \\
\hline
\end{tabular}

\section{Actividades de la vida diaria instrumentales:}

La distribución de esta variable analizada, hace referencia que el $37 \%$ de la información recopilada tiene relación con la comunicación que las personas en situación de discapacidad visual utilizan para participar en la comunidad, como es el uso de teléfono, grabadoras, entre otros. Consecutivamente la segunda actividad con mayor porcentaje de información, es movilidad en la comunidad con un total de $32,6 \%$, seguido de esto encontramos la gestión financiera con un total de $23,9 \%$, dicha información encontrada hacía referencia al uso del dinero y a la identificación de este. Adicionalmente una de las actividades que tiene menor información sobre esta área en relación con la discapacidad visual, representa cada una un $4,3 \%$ cada una (cuidado de otros, preparación de comida y limpieza, práctica en religión y mantenimiento seguridad y responder emergencias) (Tabla №5)

Tabla $N^{\mathbf{0}}$ 5: Tabla de frecuencia de las actividades de la vida diaria instrumentales relacionada con la información encontrada.

\begin{tabular}{|c|c|c|}
\hline & $\begin{array}{c}\text { Número de } \\
\text { casos }\end{array}$ & Porcentaje \\
\hline No evaluable & 1 & $2,2 \%$ \\
\hline Compras & 8 & $17,4 \%$ \\
\hline Gestión Financiera & 11 & $23,9 \%$ \\
\hline Cuidado de otros & 2 & $4,3 \%$ \\
\hline Cuidado de mascotas & 0 & $0 \%$ \\
\hline Crianza de niños & 0 & $0 \%$ \\
\hline Gestión de comunicación & 17 & $37 \%$ \\
\hline Movilidad en la comunidad & 15 & $32,6 \%$ \\
\hline $\begin{array}{c}\text { Gestión y mantenimiento de } \\
\text { salud }\end{array}$ & 3 & $6,5 \%$ \\
\hline $\begin{array}{c}\text { Preparación de comida y } \\
\text { limpieza }\end{array}$ & 2 & $4,3 \%$ \\
\hline Práctica en religión & 2 & $4,3 \%$ \\
\hline $\begin{array}{c}\text { Mantenimiento seguridad y } \\
\text { responder emergencias }\end{array}$ & 2 & $4,3 \%$ \\
\hline Todas & 6 & $13 \%$ \\
\hline Total & 69 & $100 \%$ \\
\hline
\end{tabular}




\section{Barreras:}

En relación a las sub clasificaciones de las barreras, podemos tanto como los artículos y documentos analizados describen en un $26,1 \%$ del total a las barreras de comunicación, se destaca que gran cantidad de artículos y documentos no hacían referencia a dicha variable, replicando el porcentaje anterior.
Sumado a lo anterior se encontró una nueva barrera, la cual no se está explicita dentro de la revisión bibliográfica, las barreras actitudinales sin embargo aparecen solo en un menor porcentaje que los anteriores, con un total de un $13 \%$, (Tabla $\mathrm{N}^{\circ} 6$ ).

Tabla No 6:

Tabla de frecuencia de las diversas barreras.

\begin{tabular}{|c|c|c|}
\hline & Número de casos & Porcentaje \\
\hline Barreras Arquitectónicas & 12 & $26,1 \%$ \\
\hline Barreras Urbanísticas & 4 & $8,7 \%$ \\
\hline Barreras de Transporte & 8 & $17,4 \%$ \\
\hline Barreras de Comunicación & 15 & $32,6 \%$ \\
\hline Barreras Actitudinales & 6 & $13 \%$ \\
\hline No evaluable & 15 & $32,6 \%$ \\
\hline Total & 60 & $100 \%$ \\
\hline
\end{tabular}

\section{Año:}

Durante el año 2008 se publicó la mayor cantidad de documentos con la temática a investigar, con un $17.4 \%$. En los años anteriores la información publicada presenta un porcentaje menor, manteniéndose en el tiempo (2001-2007) con un 2,2\%. Posterior al año 2008 se visualiza un incremento de las publicaciones, variando cada año. En el año 2016 se ve una disminución de la información, teniendo un porcentaje de $4,3 \%$; sin embargo, esta puede variar en el tiempo ya que solo se recopilo y analizó información hasta el primer semestre de dicho año.

Cabe destacar que un $8.7 \%$ de la información no presenta fecha de publicación o investigación. (Figura № 2). 
Figura $\mathrm{N}^{\circ} 2$ :

Distribución de información a través de los años.

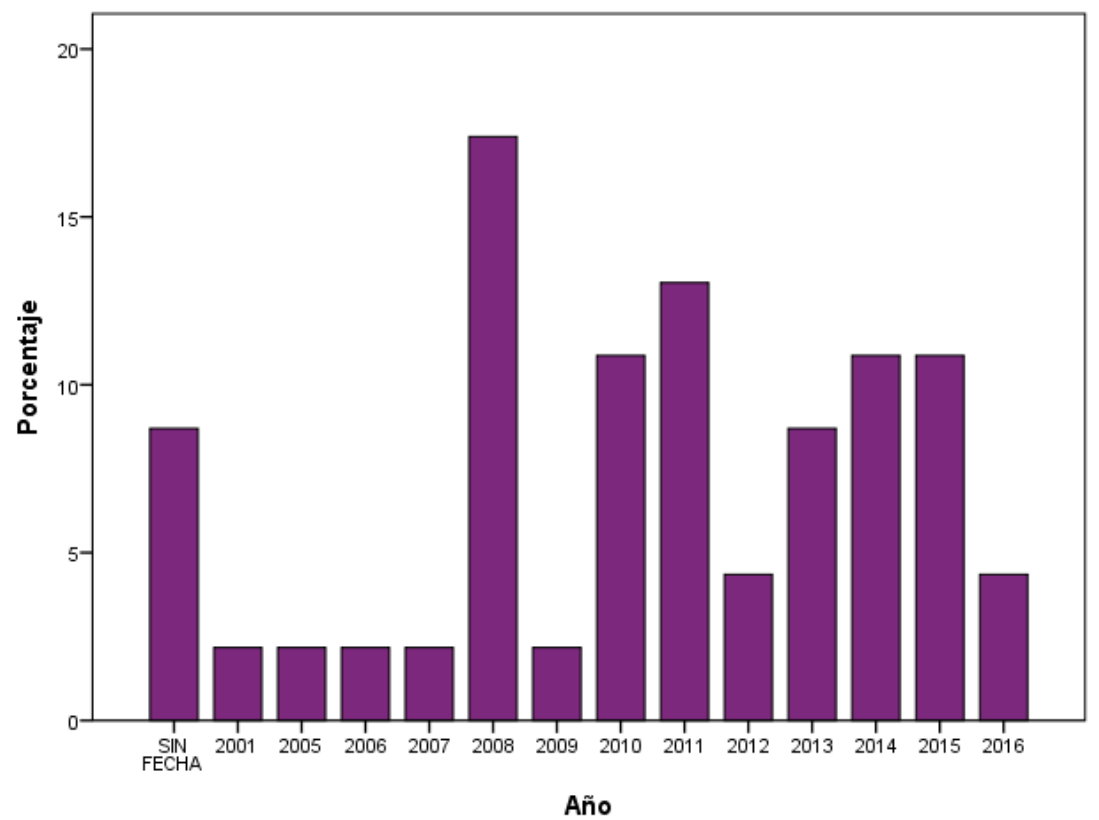

\section{DISCUSION}

De acuerdo al análisis, la mayor frecuencia de información, artículos e investigaciones encontradas fueron sobre las barreras:

Barreras de comunicación: Obstáculo principal que afecta la manera de comunicarse e informarse. Hermes (2014), señala que al implementar nuevas tecnologías, como el identificador de billetes en el entorno, mejora la inclusión social de las personas con discapacidad visual, sin embargo, estos no son accesibles para todas las personas. Es por ello que el análisis demostró que una de las mayores barreras encontradas son las de la comunicación, considerando todas las implementaciones que se han "ejecutado", por ende, podemos concluir que no han sido accesibles, ya que no se han ejecutado de forma adecuada y con su respectivo diseño universal.

Barreras arquitectónicas:_Los documentos incluyen acciones e implementos para un óptimo desempeño de las personas en situación de discapacidad visual, sin embargo, estos implementos son escasos para dicha población, ya que estas barreras están enfocadas con mayor amplitud a las personas con discapacidad física (Boudeguer, Prett y Squella, 2010).

Barreras actitudinales: Según el manual de accesibilidad esta barrera no es considerada como un obstáculo para las personas en su entorno, sin embargo de acuerdo a la información recopilada se evidencia que dicha barrera es identificada como un obstáculo para las personas, afectando en gran medida a las personas en situación de discapacidad, siendo un factor contextual, el cual impacta en el desarrollo personal y participación dentro del entorno, demostrando de esta manera que el termino discapacidad, no solo son aqueIlas deficiencias que presenta la persona, sino que el contexto en el cual se desenvuelve y esta participa, lo anterior puede restringir o facilitar su participación con el entorno y sus actividades cotidianas. (Organización Mundial de la Salud, 2016).

Según el análisis de datos, una de las actividades en que las personas son participes, es la gestión de comunicación, si bien es una actividad que les dificulta a las personas en situación de discapacidad visual, haciendo referencia a lo analizado durante la revisión ya que es la actividad con mayor porcentaje, es también 
la actividad la con mayores implementaciones de herramientas y estrategias para facilitar su ejecución, tales como sistema braille, grabadoras, audios, entre otros (Boudeguer, 2004).

Según la información analizada, aquellas AVDI específicas como cuidado de mascotas, crianza de niños, mantenimiento en salud y preparación de comida, presentan una menor frecuencia de información, considerando que no existen barreras en el entorno que dificulten el desempeño de las AVDI ya mencionadas, corroborando dicha información por la minoría de frecuencia en información encontrada en las barreras, mejorando de esta manera que las personas puedan desempeñarse de manera independiente y autónoma.

Si bien Terapia Ocupacional es una de las disciplinas del ámbito de la salud que más se preocupa de la autonomía e independencia de toda persona para desarrollar sus actividades a lo largo del ciclo vital, esta no presenta gran cantidad de información, por lo que no hay mayor conocimiento desde el área para poder complementar con otras disciplinas y mejorar la calidad de vida de estas personas. Además, de acuerdo a lo investigado, podemos referir que las personas con discapacidad visual muchas veces no logran involucrarse adecuadamente en sus AVDI, como por ejemplo, el ir de compras, acceder a la información, esto debido a las barreras existentes que limitan y obstaculizan un óptimo desempeño de las personas y a una adecuada accesibilidad, por esto mismo, estas personas se sienten incapaces de poder realizar las cosas por sí mismas, necesitando ayuda de terceros para realizar algunas tareas.

La sociedad se ha preocupado de mejorar la accesibilidad y adaptaciones para aquellas personas que presentan discapacidad física dejando de lado la discapacidad visual, es por esto que como terapeutas ocupacionales deberíamos velar por la inclusión de dicha población, mejorando su calidad de vida, fortalecer aquellas habilidades que se ven obstaculizadas por una mala accesibilidad, incorporando nuevas ayudas $u$ adaptaciones para un óptimo desempeño, favoreciendo de esta manera a que nuestra población a investigar tome decisiones de manera autónoma e independiente sin necesitar ayuda de otras personas para poder cumplir con algún objetivo dentro de sus actividades de la vida diaria instrumentales.

\section{AGRADECIMIENTOS:}

Este estudio no hubiera sido posible sin la colaboración de nuestras tutoras metodológica y disciplinar, quienes tuvieron la disposición de guiarnos dentro de nuestra investigación y apoyarnos en el proceso. Además, agradecer a la Universidad Santo Tomás, sede Concepción por permitirnos esta oportunidad de realizar un estudio que podrá colaborar para nuestra área disciplinar.

\section{REFERENCIAS BIBLIOGRÁFICAS:}

\section{CITADAS EN EL TEXTO}

Abril, J., Guerra, L., Ramírez, C., Ramón, L., \& Serrano, C. (2013). "Barreras contextuales para la la participación de las personas en discapacidad fisica, discapacidad y barreras contextuales. Recuperado el 4 de junio de 2016, de http://www.scielo.org.co/scielo. php?script=sci_arttext\&pid=S0121-08072013000100006

Algado, S. (2006). "Modelo Canadiense del desempeño ocupacional. Revista Gallega de Terapia Ocupaciona/(3). Recuperado el 11 de junio de 2016, de http://www.revistatog.com/num3/pdfs/Expertol.pdf

Álvarez, E., Gómez, S., Muñoz, I., Navarrete, E., Riveros, M., Rueda, L., \& Salgado, P. (2006). "Definición y desarrollo del concepto de Ocupación: ensayo sobre la experiencia de construcción teórica desde una identidad local". Recuperado el 9 de junio de 2016, de file:///C:/Users/Win8.1/Downloads/81-216-1-PB\%20(1).pdf

Andrade, J. (2005). "Regular el uso de perros guías, de señal o de servicio por parte de personas con discapacidad". Ley Número 20.025. Santiago, Chile. Recuperado el 2 de Mayo de 2016, de http://www. leychile.cl/Navegar?idNorma=239523

Arteaga, J. (2011). "Discapacidad visual". Recuperado el 4 de Noviembre de 2015, de http://catarina.udlap.mx/u_dl_a/tales/documentos/ Ida/arteaga_j_g/capítulo1.pdf

Ávila, A. (2010). "Marco de trabajo para la práctica de la Terapia Ocupacional; Dominio y proceso". Segunda Edición (traducción). Recuperado el 26 de Octubre de 2015, de http://www.terapia-ocupacional.com/aota2010esp.pdf

Boudeguer, A. (2004). "Transporte público accesible para personas con discapacidad en Chile: el desafío de las barreras actitudinales". Recuperado el 10 de mayo de 2016, de http://prohumana.cl/2014/02/ transporte-publico-accesible-para-personas-con-discapacidaden-chile-el-desafio-de-las-barreras-actitudinales/ 
Boudeguer, Prett y Squella. (2010). "Manual de Accesibilidad Universal". 1. Santiago, Chile. Recuperado el 4 de Marzo de 2016, de http:// www.ciudadaccesible.cl/wp-content/uploads/2012/06/manual_ accesibilidad_universal1.pdf

Caamaño, M. (2005). "Intervención del Municipio en la Política Social para las Personas con Discapacidad". Recuperado el 20 de abril de 2016, de http://www.ubiobio.cl/cps/ponencia/doc/p2.1.htm

Calvo, F. (2011). Discapacidad visual autonomía personal. Recuperado el 7 de junio de 2016, de http://sid.usal.es/idocs/F8/FDO26230/ discap_visual.pdf

Chile, G. d. (Enero de 2016). "Resultado del II Estudio Nacional de la Discapacidad en la población adulta". Recuperado el 5 de Octubre de 2016, de http://www.gob.cl/resultados-del-ii-estudio-nacionalde-la-discapacidad-en-la-poblacion-adulta/

CONADIS. (1994). "Accesibilidad de personas con movilidad reducida". Ley Número 24.314. Argentina. Recuperado el 26 de Mayo de 2016, de http://www.conadis.gov.ar/doc_publicar/access/ ley_24314.pdf

Contardo, N. (2013). "Impacto Funcional de la discapacidad visual adquirida en adultos mayores". Recuperado el 5 de junio de 2016, de http://dspace.utalca.cl:8888/salud/kinesiologia/68257.pdf

Corbalán, A. (2010). Barreras. Recuperado el 10 de mayo de 2016, de http://www.accesibilidadglobal.com/2010/07/la-definicion-deunabarrera.html

Corporacion ciudad accesible. (2016). "Endisc 2015: 1 de cada 5 chilenos vive en situación de discapacidad". Recuperado el 15 de mayo de 2016, de http://www.ciudadaccesible.cl/?p=5558

Couldrey, M., \& Herson, M. (2010). "Discapacidad y desplazamiento". Revista migraciones. Recuperado el 6 de junio de 2016, de http:// www.fmreview.org/sites/fmr/files/FMRdownloads/es/discapacidad/RMF35.pdf

De Balbinder, P. (2011). "Discapacidad visual y esquema corporal". Recuperado el 3 de Mayo de 2016, de http://www.integrando.org.ar/ investigando/dis_visual.htm

De la Fuente, M. (2010). "Normas sobre igualdad de oportunidades e inclusión social de personas con discapacidad". Ley Número 20.422(articulo 1). Santiago, Chile. Recuperado el 15 de Marzo de 2016, de http://www.leychile.cl/Navegar?idLey=20422

FinisTerrae. (2010). "Manual de accesibilidad universal". Recuperado el 26 de Octubre de 2015, de http://www.ciudadaccesible.cl/wpcontent/uploads/2012/06/manual_accesibilidad_universal1.pdf

Floyd, M., Zambrano, J., \& Antò. (2012). "Identificación de barreras del entorno que afectan la inclusion social de las personas con discapacidad motriz de miembros inferiores". Recuperado el 20 de noviembre de 2015, de http://www.scielo.org.co/pdf/sun/v28n2/ v28n2a06.pdf

FONADIS. (2004). "Informe ejecutivo VIII Region del BioBio". Recuperado el 15 de octubre de 2015, de http://www.ine.cl/canales/ chile_estadistico/encuestas_discapacidad/pdf/VIIIregion.pdf
FONADIS. (2004). "Prevalencia de personas con discapacidad en Chile". Recuperado el 15 de Noviembe de 2015, de http://www.ine. cl/canales/chile_estadistico/encuestas_discapacidad/pdf/presentacionresultadosestudionacionaldeladiscapacidad.pdf

Gomez, B., Rodriguez, F., Lamas, F., \& Pillado, P. (2010). "Ocupación y salud". 7. Recuperado el 10 de junio de 2016, de http://www. revistatog.com/suple/num6/gallegas.pdf

Hermes. (2014). "Presentan en Coquimbo dispositivo que permite identificar billetesa personas con discapacidad visual". Recuperado el 8 de mayo de 2015, de http://www.hermesnoticias.cl/?p=3374

Hernández, A. (2004). "Las personas con discapacidad, su calidad de vida y la de su entorno". Recuperado el 10 de junio de 2016, de http://www.scielo.org.co/scielo.php?script=sci_arttext\&pid $=$ S1657-59972004000100008

Hernández, M., Olivares, A., Carillo, L., Tovar, G., González, A., \& Avilés, P. (2015). "Prevalencia de trastornos visuales y su relación con la funcionalidad en adultos mayores". Recuperado el 7 de junio de 2016, de http://scielo.sld.cu/scielo.php?script=sci_arttext\&pid $=$ S0864-21762015000200005

Hinestroza, H, Roqueme, M. (2013). "Discapacidad y barreras arquitectonicas un problema de exclusión social". Recuperado el 16 de Marzo de 2016, de http://www.corposucre.edu.co/sites/default/ files/pdfs/DISCAPACIDAD\%20Y\%20BARRERAS\%20ARQUITECTONICAS\%20UN\%20PROBLEMA\%20SOCIAL.pdf

Jirón, P., \& Mansilla, P. (2013). "Atravesando la espesura de la ciudad: vida cotidiana y barreras de accesibilidad de los habitantes de la periferia urbana de Santiago de Chile". Recuperado el 3 de junio de 2016, de http://www.scielo.cl/scielo.php?pid=S071834022013000300004\&script=sci_abstract

Labrada, E. (2013). "Apropiación de las tecnologías e inclusión social de las personas con discapacidad visual". Recuperado el 2 de junio de 2016, de http://www.revista.unam.mx/vol.14/num12/art52/\#

Lago, C. (2016). "Resultados ENDISC II: 20\% de adultos con discapacidad en Chile". Recuperado el mayo de 13 de 2016, de http://blog. ciudadfacil.cl/resultados-endisc-ii-20-de-personas-con-discapacidad-en-chile/

Lorenzo, M. (2005). "Accessibility for blind and visually impaired people". Recuperado el 2 de abril de 2016, de http://www.sciencedirect.com/science/article/pii/S0531513105009660

Marchán, C y Benito, T. (2012). "La deficiencia visual". Recuperado el 22 de Octubre de 2015, de http://www.xtec.cat/ jbiayna/jjcc/arxiu/ treballs03/pdf/11.pdf

MINEDUC. (2007). "Discapacidad Visual" (Primera ed.). Recuperado el 18 de noviembre de 2015, de http://www.educacionespecial.mineduc.cl/usuarios/edu.especial/File/GuiaVisual.pdf

Organización Mundial de la Salud. (2016). Discapacidades. Recuperado el 14 de abril de 2016, de http://www.who.int/topics/disabilities/es/ 
Organización Mundial de la Salud. (2001). "Clasificacion Internacional del Funcionamiento, de la Discapacidad y la Salud". IMSERSO. Recuperado el 1 de Junio de 2016, de http://sid.usal.es/idocs/F8/ FDO4989/ciffinalb.pdf

Organización Mundial de la Salud. (2014). "Ceguera y discapacidad visual". Recuperado el 15 de mayo de 2016, de http://www.who.int/ mediacentre/factsheets/fs282/es/

Ramírez, J., \& Serrano, P. (2008). "Edad, salud y deterioro de la vision: la disminucion visual como sindrome geriatrico". Recuperado el 4 de junio de 2016, de http://www.once.es/new/servicios-especializados-en-discapacidad-visual/publicaciones-sobre-discapacidadvisual/nueva-estructura-revista-integracion/copy_of_numeros-publicados/integracion-pdf/Integracion-55.pdf

Real Academia Española. (2016). "Definición de año". Recuperado el 28 de Mayo de 2016, de http://dle.rae.es/?id=31Ws94D|31Yyrx8

Real Academia Española. (2016). "Definición de idioma". Recuperado el 28 de Mayo de 2016, de http://dle.rae.es/?id=KuMp7nw

Real Academia Española. (2016). "Definición de país". Recuperado el 28 de Mayo de 2016, de http://dle.rae.es/?id=RT371RT

Road, C. (2015). "Barreras a la participación comunes enfrentadas por las personas con discapacidades". Recuperado el 4 de junio de 2016, de http://www.cdc.gov/ncbddd/spanish/disabilityandhealth/ disability-barriers.html

Romero, M. (2007). "Actividades de la vida diaria". 23. Recuperado el 10 de junio de 2016, de http://www.um.es/analesps/v23/v23_2/1323_2.pdf

SENADIS. (2013). "Política Nacional para la Inclusión Social de las personas con discapacidad 2013-2020". Recuperado el 4 de junio de 2016, de file://C:/Users/Win8.1/Downloads/Politica\%20Nacional\%20para\%20la\%20Inclusion $\% 20$ Social\%20de $\% 20 l a s \% 20$ Personas\%20con\%20Discapacidad\%20(3).pdf

Social, M. d. (2016). "Estudio Nacional de la discapacidad 2015". Recuperado el 5 de Octubre de 2016, de http://observatorio.ministeriodesarrollosocial.gob.cl/endisc/docs/ResultadosGenerales_Endiscll_VersionExtendida_Revisada.pdf

Terapia Ocupacional. (2011). "Actividades de la vida diaria". Recuperado el 27 de octubre de 2015, de http://www.terapiaocupacionalhoy. com/2011/07/actividades-de-la-vida-diaria.html 